\title{
Confidence Estimation for Translation Prediction
}

\author{
Simona Gandrabur \\ RALI, Université de Montréal \\ gandrabu@iro. umontreal.ca
}

\author{
George Foster \\ RALI, Université de Montréal \\ foster@iro.umontreal.ca
}

\begin{abstract}
The purpose of this work is to investigate the use of machine learning approaches for confidence estimation within a statistical machine translation application. Specifically, we attempt to learn probabilities of correctness for various model predictions, based on the native probabilites (i.e. the probabilites given by the original model) and on features of the current context. Our experiments were conducted using three original translation models and two types of neural nets (single-layer and multilayer perceptrons) for the confidence estimation task.
\end{abstract}

\section{Introduction}

Most statistical models used in natural language applications are capable in principle of generating probability estimates for their outputs. However, in practice, these estimates are often quite poor and are usually interpreted simply as scores that are monotonic with probabilities. There are many contexts where good estimates of true probabilities are desirable:

- in a decision-theoretic setting, posterior probabilities are required in order to choose the lowest-cost output for a given input.

- when a collection of different models is available for some problem, output probabilities provide a principled and convenient way of combining them; and

- when multiplying conditional probabilities to compute joint distributions, the accuracy of the result is crucially dependent on the stability of the conditional estimates across different contexts-this is important for applications like speech recognition and machine translation that perform searches over a large space of output sentences, represented as sequences of words.

Given a statistical model that produces a probabilistic score, a straightforward way of obtaining a true probability is to use the score as input to another model whose output is interpreted as the desired probability. The idea is that the second model can learn how to transform the base model's score by observing its performance on new text, possibly in conjunction with other features. This approach, which is known as confidence estimation (CE), is widely used in speech recognition (Guillevic et al., 2002; Moreno et al., 2001; Sanchis et al., 2003; Stolcke et al., 1997) but is virtually unknown in other areas of natural language progessing (NLP). ${ }^{1}$

The alternatives to confidence estimation are traditional smoothing techniques such as backing off to simpler models and cross validation, along with careful marginalization and scaling where applicable to obtain the desired posterior probabilities. There is some evidence (Wessel et al., 2001) that this approach can give results that are at least as good as those obtainable with an external CE model. However, CE as we present it here is not incompatible with traditional techniques, and has several practical advantages. First, it can easily incorporate specialized features that are highly indicative of how well the base model will perform on a given input, but that may be of little use for the task of choosing the output. Since such features may be inconvenient to include in the base model, CE represents a kind of modularization, particularly as it may be possible to reuse some features for many different problems. Another advantage is that a CE layer is usually much smaller and easier to train than the baseline model; this means that it can be used to rapidly adapt a system's performance to new domains. Finally, CE typically concentrates on only the top few hy-

\footnotetext{
${ }^{1}$ A recent exception is Manmatha and Sever (2002), who describe a form of confidence estimation for combining the results of different query engines in information retrieval.
} 
potheses output by the baseline model, which is an easier task than estimating a complete distribution. This is especially true when the hypotheses of interest are drawn from a joint distribution that may be impossible in practice to enumerate.

In this paper we describe an application of confidence estimation to an interactive target-text prediction task in a translation setting, using two different types of neural nets: single-layer perceptron (SLPs) and multi-layer perceptrons (MLPs) with 20 hidden units.

The main issues that we investigate here are:

- the benefit that can be gained by using confidence estimates, in discrimination power and/or over-all application quality as computed by a simulation that estimates the benefit to the user;

- the use of different machine learning (ML) techniques for $\mathrm{CE}$;

- the relevance of various confidence features; and

- model combinations: we experiment with various model combination schemes based on the CE layer in order to improve the over-all prediction accuracy of the application.

Among the more interesting results we will present are the comparisons between the discrimination capacity of the native probabilities and the probabilities of correctness produced by the CE layer. Depending on the underlying SMT model, we obtained a relative improvement in correct rejection rate (CR) ranging from $3.90 \%$ to $33.09 \%$ at a fixed 0.80 correct acceptance rate (CA) for prediction lengths of up to four words. We also measured relative improvements of approximately $10 \%$ in estimated benefit to the user with our application.

In the following section we briefly describe the text prediction application we are aiming to improve. Next we outline the CE approach and the evaluation methods we applied. Finally, we report the results obtained in our experiments and conclude with suggestions for future work.

\section{Text Prediction for Translators}

The application we are concerned with in this paper is an interactive text prediction tool for translators. The system observes a translator in the process of typing a target text and, after every character typed, has the opportunity to display a suggestion about what will come next, based on the source sentence under translation and the prefix of its translation that has already been typed. The translator may incorporate suggestions into the text if they are helpful, or simply ignore them and keep typing.

Suggestions may range in length from 0 characters to the end of the target sentence; it is up to the system to decide how much text to predict in a given context, balancing the greater potential benefit of longer predictions against a greater likelihood of being wrong, and a higher cost to the user (in terms of distraction and editing) if they are wrong or only partially right.

Our solution to the problem of how much text to predict is based on a decision-theoretic framework in which we attempt to find the prediction that maximizes the expected benefit to the translator in the current context (Foster et al., 2002b). Formally, we seek:

$$
\hat{\mathbf{x}}=\underset{\mathbf{x}}{\operatorname{argmax}} B(\mathbf{x} \mid \mathbf{h}, \mathbf{s}),
$$

where $\mathbf{x}$ is a prediction about what will follow $\mathbf{h}$ in the translation of a source sentence $\mathbf{s}$, and $B(\mathbf{x} \mid \mathbf{h}, \mathbf{s})$ is the expected benefit in terms of typing time saved. As described in (Foster et al., 2002b), $B\left(\hat{\mathbf{x}}_{m} \mid \mathbf{h}, \mathbf{s}\right)=$ $\sum_{k=0}^{l} p(k \mid \mathbf{x}, \mathbf{h}, \mathbf{s}) B(\mathbf{x} \mid \mathbf{h}, \mathbf{s}, k)$ depends on two main quantities: the probability $p(k \mid \mathbf{x}, \mathbf{h}, \mathbf{s})$ that exactly $k$ characters from the beginning of $\mathbf{x}$ are correct, and the benefit $B(\mathbf{x} \mid \mathbf{h}, \mathbf{s}, k)$ to the translator if this is the case. $B(\mathbf{x} \mid \mathbf{h}, \mathbf{s}, k)$ is estimated from a model of user behaviour-based on data collected in user trials of the tool-that captures the cost of reading a prediction and performing any necessary editing, as well as the somewhat random nature of people's decisions to accept. Prediction probabilities $p(k \mid \mathbf{x}, \mathbf{h}, \mathbf{s})$ are derived from a statistical translation model for $p(w \mid \mathbf{h}, \mathbf{s})$, the probability that some word $w$ will follow the target text $\mathbf{h}$ in the translation of a source sentence $\mathbf{s}$.

Because optimizing (1) directly is expensive, we use a heuristic search procedure to approximate $\hat{\mathbf{x}}$. For each length $m$ from 1 to a fixed maximum of $M$ (4 in this paper), we perform a Viterbi-like beam search with the translation model to find the sequence of words $\hat{\mathbf{w}}_{m}=$ $w_{1}, \ldots, w_{m}$ most likely to follow $\mathbf{h}$. For each such sequence, we form a corresponding character sequence $\hat{\mathbf{x}}_{m}$ and evaluate its benefit $B\left(\hat{\mathbf{x}}_{m}, \mathbf{h}, \mathbf{s}\right)$. The final output is the prediction $\hat{\mathbf{x}}_{m}$ with maximum benefit, or nothing if all benefit estimates are negative.

To evaluate the system, we simulate a translator's actions on a given source text, using an existing translation as the text the translator wishes to type, and the user model to determine his or her responses to predictions and to estimate the resulting benefit. Further details are given in (Foster et al., 2002b).

\subsection{Translation Models}

We experimented with three different translation models for $p(w \mid \mathbf{h}, \mathbf{s})$. All have the property of being fast enough to support real-time searches for predictions of up to 5 words.

The first model, referred to as Maxent1 below, is a loglinear combination of a trigram language model with a 
maximum entropy translation component that is an ana$\log$ of the IBM translation model 2 (Brown et al., 1993). This model is described in (Foster, 2000). Its major weakness is that it does not keep track of which words in the current source sentence have already been translated, and hence it is prone to repeating previous suggestions. The second model, called Maxent 2 below, is similar to Maxent1 but with the addition of extra parameters to limit this behaviour (Foster et al., 2002a).

The final model, called Bayes below, is also described in (Foster et al., 2002a). It is a noisy-channel combination of a trigram language model and an IBM model 2 for the source text given target text. This model has roughly the same theoretical predictive capability as Maxent2, but unlike the Maxent models it is not discriminatively trained, and hence its native probability estimates tend to be much worse than theirs.

\subsection{Computing Smoothed Conditional Probabilities}

In order to calculate the character-based probabilities $p(k \mid \mathbf{x}, \mathbf{h}, \mathbf{s})$ required for estimating expected benefit, we need to know the conditional probabilities $p\left(w \mid w_{1}, \ldots, w_{i-1}, \mathbf{h}, \mathbf{s}\right)$ that some word $w$ will follow $w_{1}, \ldots, w_{i-1}$ in the context $(\mathbf{h}, \mathbf{s})$. These are derived from correctness estimates obtained from our confidenceestimation layer as follows. As explained below, estimates from the $\mathrm{CE}$ layer are in the form $p(C=$ $\left.1 \mid \hat{\mathbf{w}}_{m}, \mathbf{h}, \mathbf{s}\right)$, where $\hat{\mathbf{w}}_{m}$ is the most probable prediction of length $m$ according to the base translation model. Define a smoothed joint distribution over predictions of length $m$ as:

$$
p_{s}\left(\mathbf{w}_{m} \mid \mathbf{h}, \mathbf{s}\right)= \begin{cases}p\left(C=1 \mid \hat{\mathbf{w}}_{m}, \mathbf{h}, \mathbf{s}\right), & \mathbf{w}_{m}=\hat{\mathbf{w}}_{m} \\ p\left(\mathbf{w}_{m} \mid \mathbf{h}, \mathbf{s}\right) / z_{m}, & \text { else }\end{cases}
$$

where $p\left(\mathbf{w}_{m} \mid \mathbf{h}, \mathbf{s}\right)=\prod_{i=1}^{m} p\left(w_{i} \mid w_{1}, \ldots, w_{i-1}, \mathbf{h}, \mathbf{s}\right)$ is calculated from the conditional probabilities given by the base model; and

$$
z_{m}=\frac{1-p\left(\hat{\mathbf{w}}_{m} \mid \mathbf{h}, \mathbf{s}\right)}{1-p\left(C=1 \mid \hat{\mathbf{w}}_{m}, \mathbf{h}, \mathbf{s}\right)}
$$

is a normalization factor. Then the required smoothed conditional probabilities are estimated from the smoothed joint distributions in a straightforward way:

$$
p_{s}\left(w \mid w_{1}, \ldots, w_{i-1}, \mathbf{h}, \mathbf{s}\right)=\frac{p_{s}\left(w_{1}, \ldots, w_{i-1}, w \mid \mathbf{h}, \mathbf{s}\right)}{p_{s}\left(w_{1}, \ldots, w_{i-1} \mid \mathbf{h}, \mathbf{s}\right)}
$$

where $p\left(w_{1}, \ldots, w_{i-1} \mid \mathbf{h}, \mathbf{s}\right) \equiv 1$ when $i=1$.

\section{Confidence Estimation with Neural Nets}

Our approach for CE consists in training neural nets to estimate the conditional probability of correctness $p(C=$ $\left.1 \mid \hat{\mathbf{w}}_{m}, \mathbf{h}, \mathbf{s},\left\{\mathbf{w}_{m}^{1}, \ldots, \mathbf{w}_{m}^{n}\right\}\right)$, where $\hat{\mathbf{w}}_{m}=\mathbf{w}_{m}^{1}$ is the most probable prediction of length $m$ from a $n$-best set of alternative predictions according to the base model. In our experiments the prediction length $m$ varies between 1 and 4 and $n$ is at most 5 . As the n-best predictions $\left\{\mathbf{w}_{m}^{1}, \ldots, \mathbf{w}_{m}^{n}\right\}$ are themselves a function of the context, we will simply note the conditional probability of correctness by $p\left(C=1 \mid \hat{\mathbf{w}}_{m}, \mathbf{h}, \mathbf{s}\right)$.

We experimented with two types of neural nets: singlelayer perceptrons (SLPs) and multi-layer perceptrons (MLPs) with 20 hidden units. For both, we used a softmax activation function and gradient descent training with a negative log-likelihood error function. Given suitably-behaved class-conditional feature distributions, this setup is guaranteed to yield estimates of the true posterior probabilities $p\left(C=1 \mid \hat{\mathbf{w}}_{m}, \mathbf{h}, \mathbf{s}\right)$ (Bishop, 1995).

\subsection{Single Layer Neural Nets and Maximum Entropy Models}

It is interesting to note the relation between the SLP and maximum entropy models. For the problem of estimating $p(y \mid \mathbf{x})$ for a set of classes $y$ over a space of input vectors $\mathbf{x}$, a single-layer neural net with "softmax" outputs takes the form:

$$
p(y \mid \mathbf{x})=\exp \left(\vec{\alpha}_{y} \cdot \mathbf{x}+b\right) / Z(\mathbf{x})
$$

where $\vec{\alpha}_{y}$ is a vector of weights for class $y, b$ is a bias term, and $Z(\mathbf{x})$ is a normalization factor, the sum over all classes of the numerator. A maximum entropy model is a generalization of this in which an arbitrary feature function $\mathbf{f}_{y}(\mathbf{x})$ is used to transform the input space as a function of $y$ :

$$
p(y \mid \mathbf{x})=\exp \left(\vec{\alpha} \cdot \mathbf{f}_{y}(\mathbf{x})\right) / Z(\mathbf{x}) .
$$

Both models are trained by maximum likelihood methods. Given $C$ classes, the maximum entropy model can simulate a SLP by dividing its weight vector into $C$ blocks, each the size of $\mathbf{x}$, then using $\mathbf{f}_{y}(\mathbf{x})$ to pick out the $y$ th block:

$$
\mathbf{f}_{y}(\mathbf{x})=\left(\mathbf{0}_{1}, \ldots, \mathbf{0}_{y-1}, \mathbf{x}, \mathbf{0}_{y+1}, \ldots, \mathbf{0}_{C}, 1\right),
$$

where each $\mathbf{0}_{i}$ is a vector of 0 's and the final 1 yields a bias term.

The advantage of maximum-entropy models is that their features can depend on the target class. For naturallanguage applications where target classes correspond to words, this produces an economical and powerful representation. However, for CE, where the output is binary (correct or incorrect), this capacity is less interesting. In fact, there is no a priori reason to use a different set of features for correct outputs or incorrect ones, so the natural form of a maxent model for this problem is identical to a SLP (modulo a bias term). Therefore the experiments we describe below can be seen as a comparison between maxent models and neural nets with a hidden layer. 


\subsection{Confidence Features}

The features we use can be divided into three families: ones designed to capture the intrinsic difficulty of the source sentence $\mathbf{s}$ (for any NLP task); ones intended to reflect how hard $\mathbf{s}$ is to translate in general, and ones intended to reflect how hard $\mathbf{s}$ is for the current model to translate. For the first two families, we used two sets of values: static ones that depend on s; and dynamic ones that depend on only those words in s that are deemed to be still untranslated, as determined by an IBM2 word alignment between $\mathbf{s}$ and $\mathbf{h}$. The features are:

- family 1: trigram perplexity, minimum trigram word probability, average word frequency, average word length, and number of words;

- family 2: average number of translations per source word (according to an independent IBM1), average IBM1 source word entropy, number of source tokens still to be translated, number of unknown source tokens, ratio of linked to unlinked source words within the aligned region of the source sentence, and length of the current target-text prefix; and

- family 3: average number of search hypotheses pruned (ie outside the beam) per time step, final search lattice size, active vocabulary size (number of target words considered in the search), number of nbest hypotheses, rank of current hypothesis, probability ratio of best hypothesis to sum of top 5 hypotheses, and base model probability of current prediction.

\section{Evaluation}

Evaluation is performed using test sets of translation predictions, each tagged as correct or incorrect. A translation prediction $\mathbf{w}_{m}$ is tagged as correct if and only if an identical word sequence is found in the reference translation, properly aligned. This reflects our application, where we attempt to match what a particular translator has in mind, not simply produce any correct translation. We use two types of evaluation methods: ROC curves and a user simulation as described above.

\subsection{ROC curves}

Consider a set of tokens $t_{i} \in D$ from given domain $D$. Each token $t_{i}$ is labelled with a tag $\mathrm{C}\left(t_{i}\right)=1$ if it is considered correct or $\mathrm{C}\left(t_{i}\right)=0$ if it is false. Consider a function $s: D \rightarrow[a, b]$ that associates a confidence score $s(t) \in[a, b]$ to any token $t_{i} \in D . s$ is not necessarily a probability, it can range over any real interval $[a, b]$.

Given a rejection threshold $\theta \in[a, b]$, any token $t_{i} \in$ $D$ is rejected if $s\left(t_{i}\right)<\theta$ and it is accepted otherwise. The correct acceptence rate $\mathrm{CA}(\theta)$ of a threshold $\theta$ over
$D$ is the rate of correct tokens $t_{i} \in D$ with $s\left(t_{i}\right) \geq \theta$. That is:

$$
\mathrm{CA}(\theta)=\frac{\left|\left\{t_{i} \in D \mid \mathrm{C}\left(t_{i}\right)=1 \wedge s\left(t_{i}\right) \geq \theta\right\}\right|}{\left|\left\{t_{i} \in D \mid \mathrm{C}\left(t_{i}\right)=1\right\}\right|} .
$$

Similarly, the correct rejection rate $\operatorname{CR}(\theta)$ is the rate of false tokens $t_{i}$ such that $s\left(t_{i}\right)<\theta$ :

$$
\mathrm{CR}(\theta)=\frac{\left|\left\{t_{i} \in D \mid \mathrm{C}\left(t_{i}\right)=0 \wedge s\left(t_{i}\right)<\theta\right\}\right|}{\left|\left\{t_{i} \in D \mid \mathrm{C}\left(t_{i}\right)=0\right\}\right|} .
$$

As $\theta$ ranges over $[a, b]$, the value pairs $(\mathrm{CA}(\theta), \operatorname{CR}(\theta)) \in[0,1] \times[0,1]$ define a curve, called the ROC curve of $s$ over $D$. The discrimination capacity of $s$ is given by its capacity to distinguish correct from false tokens. Consequently, a perfect ROC curve would describe the square $(0,1),(1,1),(1,0)$. This is the case whenever there exists a threshold $\theta \in[a, b]$ that separates all correct tokens in $D$ from all the false ones, meaning that the score ranges of correct, respectively false, tokens don't overlap. The worst case scenario, describing a scoring function that is completely irrelevent for correct/false discrimination, corresponds to the diagonal $(0,1),(1,0)$. Note that the inverse of the ideal ROC curve, the plot overlapping the axes $(1,0),(0,0),(1,0)$ is equivalent to its inverse from a discrimination capacity point of view: it suffices to invert the rejection algorithm by accepting all tokens that have a score inferior to the rejection threshold.

In our setting, the tokens are the $\hat{\mathbf{w}}_{m}$ translation predictions and the score function is the conditional probability $p\left(C=1 \mid \hat{\mathbf{w}}_{m}, \mathbf{h}, \mathbf{s}\right)$.

In order to easily compare the discrimination capacity of various scoring functions we use a raw measure, the integral of the ROC curve, or IROC. A perfect ROC curve will have an IROC $=1.0$ (respectively 0.0 in the inverse case). The worst case scenario corresponds to an IROC of 0.5. We also compare various scoring functions by fixing an operational point at $C A=0.80$ and observing the corresponding $C R$ values.

\section{Experimental Set-up}

The data for our experiments originates from the Hansard English-French parallel corpus. In order to generate the train and test sets, we use 1.3 million (900000 for training and 400000 for testing purposes) translation predictions for each fixed prediction length of one, two, three and four words, summing to a total of 5.2 million prediction examples. Each original SMT model experiment was combined with two different CE model architectures: MLPs with one hidden layer containing 20 hidden units and SLP (sometimes also referred to as MLPs with 0 hidden units). Moreover, for each (native model, CE model architecture)-pair, we train five separate CE models: one 


\begin{tabular}{|l|cc|}
\hline \multicolumn{3}{|c|}{ Bayes: $m=1, \ldots, 4, \mathrm{CA}=0.80$} \\
\hline Model & IROC & CR \\
\hline native probability & 0.8019 & 0.6604 \\
SLP & 0.8357 & 0.7211 \\
MLP & 0.8679 & 0.7728 \\
\hline
\end{tabular}

Table 1: Comparison of discrimination capacity between the Bayes prediction model probability and the CE of the corresponding SLP and MLP on predictions of up to four words

for each fixed prediction length of one, two, three or four words, and an additional model for variable prediction lengths of up to four words. ${ }^{2}$

\section{ROC Evaluations}

In this section we report the ROC evaluation results. The user-model evaluation results are presented in the following section.

\subsection{CE and Native SMT Probabilites}

The first question we wish to address is whether we can improve the correct/false discrimination capacity by using the propability of correctness estimated by the $\mathrm{CE}$ model instead of the native probabilites.

For each SMT model we compare the ROC plots, IROC and CA/CR values obtained by the native probability and the estimated probability of correctness output by the corresponding SLPs (also noted as mlp-0-hu) and the 20 hidden units MLPs on the one-to-four word prediction task.

Results obtained for various length predictions of up to four words using the Bayes models are summarized in figure (1)and in table 1 below, and are encouraging. At a fixed CA of 0.80 we obtain CR increases from 0.6604 for the native probability to 0.7211 for the SLP and 0.7728 for the MLP. The over-all gain is also evident from the the relative improvements in IROC obtained by the SLP and MLP models over the native probability, that are respectively $17.06 \%$ and $33.31 \%$. These results are quite significant.

Note that the improvements obtained in the fixedlength 4-word-prediction tasks with the Bayes model (figure (2) and table 2) model are even larger: the relative improvements on IROC are $32.36 \%$ and $50.07 \%$ for the SLP and the MLP, respectively.

However, the results obtained in the Maxent models are much less positive: the SLP CR actually drops, while the MLP CR only increases slightly to a $4.80 \%$ relative

\footnotetext{
${ }^{2}$ Training and testing of the neural nets was done using the open-source Torch toolkit ((Collobert et al., 2002), http://www.torch.ch/), which provides efficient C++ implementations of many ML algorithms.
}

Figure 1: Bayes: $m=1, \ldots, 4$

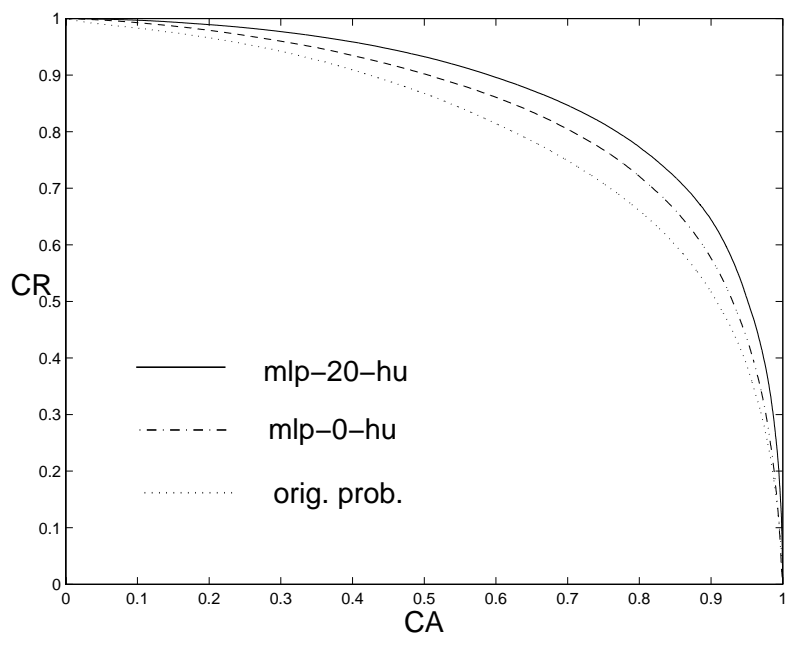

\begin{tabular}{|l|cc|}
\hline \multicolumn{3}{|c|}{ Bayes: $m=4, \mathrm{CA}=0.80$} \\
\hline Model & IROC & $\mathrm{CR}$ \\
\hline native probability & 0.7281 & 0.4998 \\
SLP & 0.8161 & 0.6602 \\
MLP & 0.8560 & 0.7503 \\
\hline
\end{tabular}

Table 2: Comparison of discrimination capacity between the Bayes prediction model probability and the CE of the corresponding SLP and MLP on fixed-length predictions of four words

Figure 2: Bayes: $m=4$

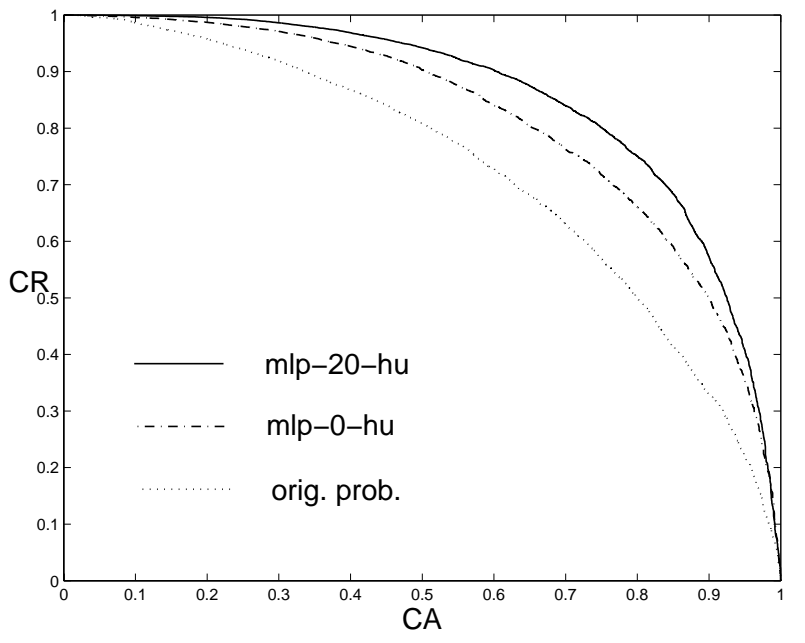




\begin{tabular}{|l|cc|}
\hline \multicolumn{3}{|l|}{ Maxent1: $m=1, \ldots, 4, \mathrm{CA}=0.80$} \\
\hline Model & IROC & CR \\
\hline native probability & 0.8581 & 0.7467 \\
SLP & 0.8401 & 0.7142 \\
MLP & 0.8636 & 0.7561 \\
\hline
\end{tabular}

Table 3: Comparison of discrimination capacity between the Maxent 1 prediction model probability and the $\mathrm{CE}$ of the corresponding SLP and MLP on predictions of up to four words

\begin{tabular}{|l|cc|}
\hline \multicolumn{3}{|c|}{ Maxent2: $m=1, \ldots, 4, \mathrm{CA}=0.80$} \\
\hline Model & IROC & CR \\
\hline native probability & 0.8595 & 0.7479 \\
SLP & 0.8352 & 0.6973 \\
MLP & 0.8638 & 0.7599 \\
\hline
\end{tabular}

Table 4: Comparison of discrimination capacity between the Maxent2 prediction model probability and the CE of the corresponding SLP and MLP on predictions of up to four words

improvement in the CR rate for the Maxent 1 model ( table 3) and only $3.9 \%$ for the Maxent 2 model ( table 4). The results obtained with the two Maxent models are very similar. We therefore only draw the ROC curve for the Maxent2 model (figure (3).

It is interesting to note that the native model prediction accuracy didn't affect the discrimination capacity of the corresponding probability of correctness of the CE models. This result is illustrated in table below, where $\% C=1$ is the percentage of correct predictions. Even though the Bayes' model accuracy and IROC is significantly lower then the Maxent model's, the CE IROC values are almost identical.

\subsection{Relevance of Confidence Features}

We investigated the relevance of different confidence features by using the IROC values of single-feature models for the 1-4 word prediction task, with both Maxent 1 and Bayes base models.

The group of features that performs best over both models are the model- and search-dependent features described above, followed by the features that capture the intrinsic difficulty of the source sentence and the targetprefix. Least valuable are the remaining features that capture translation difficulty. The single most significant feature is native probability, followed by the probability ratio of the best hypothesis, and the prediction length. Somewhat unsurprisingly, the weaker Bayes models are much more sensitive to longer translations than the Maxent models.
Figure 3: Maxent2: $m=1, \ldots, 4$

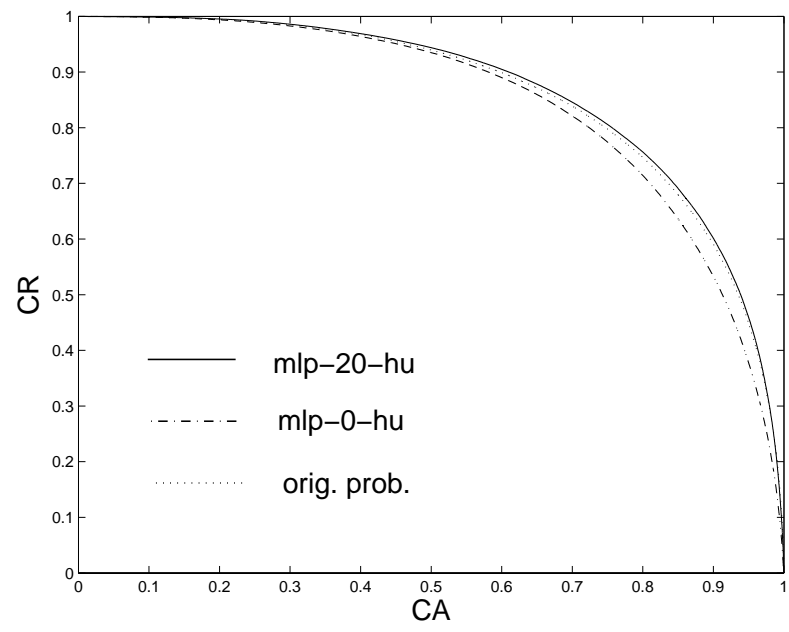

\begin{tabular}{|l|ccc|}
\hline \multicolumn{4}{|c|}{ Discrimination vs. prediction length: Maxent2 } \\
\hline $\begin{array}{l}\text { Prediction } \\
\text { length } m\end{array}$ & $\% \mathrm{C}=1$ & $\begin{array}{c}\text { IROC native } \\
\text { probability }\end{array}$ & $\begin{array}{c}\text { IROC CE } \\
\text { MLP }\end{array}$ \\
\hline$m=1$ & 44.78 & 0.7926 & 0.7986 \\
$m=2$ & 23.30 & 0.8074 & 0.8121 \\
$m=3$ & 13.12 & 0.8261 & 0.8245 \\
$m=4$ & 7.74 & 0.8517 & 0.8567 \\
$m=1, \ldots, 4$ & 22.23 & 0.8595 & 0.8638 \\
\hline
\end{tabular}

Table 5: Impact of prediction length on discrimination capacity and accuracy for the Maxent 2 prediction model

\subsection{Dealing with predictions of various lengths}

We compared different approaches for dealing with various length predictions: we trained four separate MLPs for fixed length predictions of one through four words; and a single MLP over predictions of varying lengths. Results are given in table 5 and figure (4)

\section{Model Combination}

In this section we describe how various model combinations schemes affect prediction accuracy. We use the Bayes and the Maxent2 prediction models: we try to exploit the fact that these two models, being fundamentally different, tend to be complementary in some of their responses. The CE models we use are the corresponding MLPs, as they clearly outperform the SLPs. The results presented in table 6 are reported on the variable-length prediction task for up to four words.

The combination schemes are the following: we run the two prediction models in parallel and choose one of the proposed prediction hypotheses according to the following voting criteria:

- Maximum CE vote: choose the prediction with the highest CE; 
Figure 4: Maxent2: $m=1, m=2, m=3, m=4, m=$ $1, \ldots, 4$

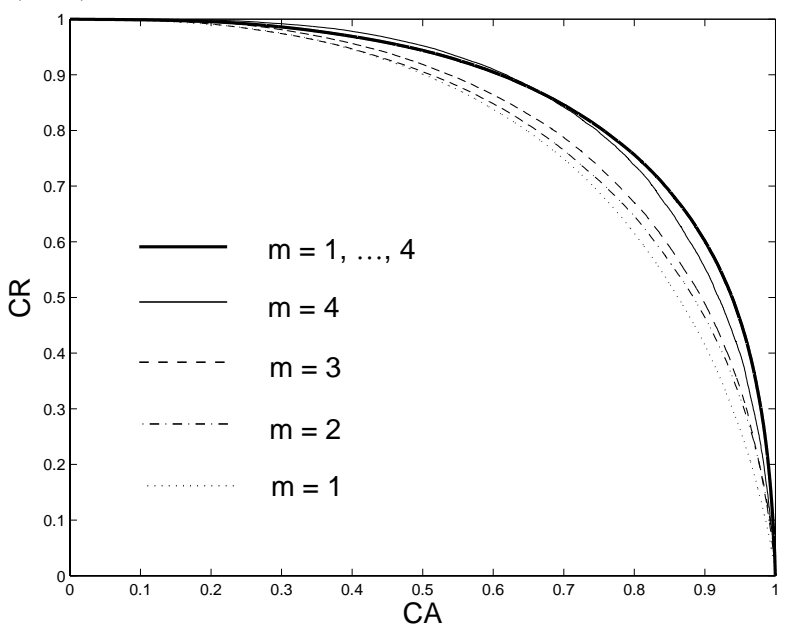

Model Combination Prediction Accuracy

\begin{tabular}{|l|c|}
\hline Prediction model combination & Accuracy \\
\hline Bayes alone & 8.77 \\
Maxent alone & 22.23 \\
Max native probability vote combination & 17.49 \\
Max CE vote combination & 23.86 \\
Optimal combination & 27.79 \\
\hline
\end{tabular}

Table 6: Prediction accuracy of the Bayes and Maxent2 model compared with combined model accuracy

- Maximum native probability vote: choose the prediction with the highest native probability.

As a baseline comparison, we use the accuracy of the individual native prediction models. Then we compute the maximum gain we can expect with an optimal model combination strategy, obtained by running an "oracle" that always picks the right answer.

The results are very positive: the maximum CE voting scheme obtains a $29.31 \%$ of the maximum possible accuracy gain over the better of the two indiviual models (Maxent2). Moreover, if we choose the maximum native probability vote, the overall accuracy actually drops significantly. These results are a strong motivation for our post-prediction confidence estimation approach: by training an additional $\mathrm{CE}$ layer using the same confidence features and training data for different underlying prediction models we obtain more uniform estimates of the probability of correctness.

\section{User-Model Evaluations}

As described in section 2, we evaluated the prediction system as a whole by simulating the actions of a translator on a given source text and measuring the gain

\begin{tabular}{|l|r|r|r|r|r|}
\hline model & base & mults & SLP & MLP & best \\
\hline Bayes & 3.2 & 6.5 & 6.4 & 6.4 & 11.8 \\
ME1 & 16.6 & 16.5 & 18.1 & 18.3 & 23.5 \\
ME2 & 17.4 & 17.4 & 19.0 & 19.3 & 24.3 \\
\hline
\end{tabular}

Table 7: Percentage of typing time saved for various CE configurations.

with a user model. In order to abstract away from approximations made in deriving character-based probabilities $p(k \mid \mathbf{x}, \mathbf{h}, \mathbf{s})$ used in the benefit calculation from word-based probabilities, we employed a specialized user model. In contrast to the realistic model described in (Foster et al., 2002b), this assumes that users accept predictions only at the beginnings of words, and only when they are correct in their entirety. To reduce variation further, it also assumes that the user always accepts a correct prediction as soon as it is suggested. Thus the model's estimates of benefit to the user may be slightly overoptimistic: the limited opportunities for accepting and editing must be balanced against the user's inhumanly perfect decision-making. However, its main purpose is not realism but simply to allow for a fair comparison between the base and the CE models.

Simulations with all three translation models were performed using a 500-sentence test text. At each prediction point, the benefits associated with best predictions of 1-4 words in length were compared to decide which (if any) to propose. The results, in terms of percentages of typing time saved, are shown in table 8: base corresponds to the base model; mults to length-specific probability multipliers tuned to optimize benefit on a held-out corpus; SLP and $M L P$ to CE estimates; and best to using an oracle to pick the length that maximizes benefit.

Although the CE layer provides no gain over the much simpler probability-multiplier approach for the Bayes model, the gain for both maxent models is substantial, around $10 \%$ in relative terms and $25 \%$ of the theoretical maximum gain (over the base model) with the MLP and slightly lower with the SLP.

\section{Conclusion}

The results obtained in this paper can be summarized in the following set of questions and answers:

- Can the probabilities of correctness estimated by the CE layer exceed the native probablities in discrimination capacity? Depending on the underlying SMT model, we obtained a relative improvement in correct rejection rate (CR) ranging from $3.90 \%$ to $33.09 \%$ at a fixed 0.80 (CA) correct acceptance rate for prediction lengths varying between 1 and 4 .

- Can we improve the overall performance of the un- 
derlying SMT application using confidence estimation? In simulated results, we found a significant gain (10\% relative) in benefit to a translator due to the use of a CE layer in two of three translation models tested.

- Can prediction accuracy of the application be improved using prediction model combinations? A maximum CE voting scheme yields a $29.31 \%$ accuracy improvement of the maximum possible accuracy gain. A similar voting scheme using native probabilies significantly decreases the accuracy of the model combination.

- How does the prediction accuracy of the native models influence the CE accuracy? Prediction accuracy didn't prove to be a significant factor in determining the discrimination capacity of the confidence estimate.

- How does CE accuracy change with various ML approches? A multi-layer perceptron (MLP) with 20 hidden units significantly outperformed one with 0 hidden units (equivalent to a maxent model for this application).

- Confidence feature selection: which confidence features are more useful and how does their discrimination capacity vary with different contexts and different native SMT models? Confidence features based on the original model and the n-best prediction turned out to be the most relevant group of featured, folowed by features that capture the intrinsic difficulty of the source text and finally translationdifficulty-specific features. We also observed interesting variations in relevance as the original models changed.

Future work will include the search for more relevant confidence features, such as features based on consenus over word-lattices ((Mangu et al., 2000)), past performance, the use of more appropriate correct/false tagging methods and experiments with different machine learning techniques. Finally, we would like to investigate whether confidence estimation can be used to improve the model prediction accuray, either by using re-scoring techniques or using the confidence estimates during search (decoding).

\section{References}

Christopher M. Bishop. 1995. Neural Networks for Pattern Recognition. Oxford.

Peter F. Brown, Stephen A. Della Pietra, Vincent Della J. Pietra, and Robert L. Mercer. 1993. The mathematics of Machine Translation: Parameter estimation. Computational Linguistics, 19(2):263-312, June.

R. Collobert, S. Bengio, and J. Mariéthoz. 2002. Torch: a modular machine learning software library. Technical Report IDIAP-RR 02-46, IDIAP.

George Foster, Philippe Langlais, and Guy Lapalme. 2002a. Text prediction with fuzzy alignments. In Stephen D. Richardson, editor, Proceedings of the 5th Conference of the Association for Machine Translation in the Americas, Tiburon, California, October. Springer-Verlag.

George Foster, Philippe Langlais, and Guy Lapalme. 2002b. User-friendly text prediction for translators. In Proceedings of the 2002 Conference on Empirical Methods in Natural Language Processing (EMNLP), Philadelphia, PA.

George Foster. 2000. Incorporating position information into a Maximum Entropy / Minimum Divergence translation model. In Proceedings of the 4th Computational Natural Language Learning Workshop (CoNLL), Lisbon, Portugal, September. ACL SigNLL.

Didier Guillevic, Simona Gandrabur, and Yves Normandin. 2002. Robust semantic confidence scoring. In Proceedings of the 7th International Conference on Spoken Language Processing (ICSLP) 2002, Denver, Colorado, September.

L. Mangu, E. Brill, and A. Stolcke. 2000. Finding consensus in speech recognition: word error minimization and other applications of confusion networks. Computer Speech and Language, 14(4):373-400.

R. Manmatha and H. Sever. 2002. A formal approach to score normalization for meta-search. In M. Marcus, editor, Proceedings of HLT 2002, Second International Conference on Human Language Technology Research, pages 98-103, San Francisco. Morgan Kaufmann.

P. Moreno, B. Logan, and B. Raj. 2001. A boosting approach for confidence scoring. In Eurospeech.

A. Sanchis, A. Juan, and E. Vidal. 2003. A simple hybrid aligner for generating lexical correspondences in parallel texts. In ICASSP 2003, pages 29-35.

A. Stolcke, Y. Koenig, and M. Weintraub. 1997. Explicit word error minimization in $\mathrm{n}$-best list rescoring. In Proc. 5th Eur. Conf. Speech Communication and Technology, volume 1, pages 163-166.

Frank Wessel, Ralf Schlüter, Klaus Macherey, and Hermann Ney. 2001. Confidence measures for large vocabulary continuous speech recognition. IEEE Transactions on Speech and Audio Processsing, 9(3):288298. 